EXTENDED REPORT

\title{
Vascular leucocyte adhesion molecules unaltered in the human retina in diabetes
}

\author{
J M Hughes, A Brink, A N Witmer, M Hanraads-de Riemer, I Klaassen, R O Schlingemann
}

Br J Ophthalmol 2004;88:566-572. doi: 10.1136/bjo.2003.021204

See end of article for authors' affiliations

.....................

Correspondence to:

Dr R O Schlingemann,

Department of

Ophthalmology, Academic

Medical Center,

Meibergdreef 15, 1105

AZ Amsterdam,

Netherlands;

r.schlingemann@

amc.uva.nl

Accepted for publication

1 August 2003
Background/aims: Capillary occlusion is believed to have a critical role in the development of diabetic retinopathy (DR). The exact mechanism by which it occurs, however, remains unclear. Several in vitro and animal model studies have suggested increased adhesion of leucocytes to the endothelium via upregulated ICAM-1 on the retinal microvasculature as a possible mechanism. In this comparative immunohistochemical study the expression of ICAM- 1 was compared in the retinal vasculature of 41 eyes obtained from 37 diabetic people with 19 eyes from 19 non-diabetic controls.

Methods: Serial cryosections of postmortem posterior tissue from 41 diabetic eyes and 19 non-diabetic eyes were stained with the monoclonal antibodies ICAM-1 (two clones), CD31 (panendothelial marker), and PAL-E (vascular leakage marker).

Results: A similar pattern of vascular ICAM-1 staining was observed between diabetic and non-diabetic eyes. A diffuse ICAM-1 staining of the retina was also observed that was significantly more intense in the diabetic subjects $(p=0.001)$.

Conclusion: These results indicate that ICAM-1 is constitutively expressed on retinal and choroidal vasculature of non-diabetic, control subjects and that this level of expression is not significantly altered by the diabetic environment. Taken together, these results do not support the prevalent paradigm of increased adhesion molecule expression as a primary mechanism responsible for capillary occlusion reported in diabetic individuals.

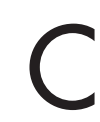
apillary non-perfusion is a hallmark of diabetic retinopathy (DR) which with time develops in nearly all people with diabetes. ${ }^{1}$ Capillary closure is considered a potentially critical event in the progression of DR to its more detrimental forms, proliferative diabetic retinopathy, in which neovascularisation occurs, and ischaemic diabetic maculopathy. ${ }^{2}$ The mechanisms by which capillary nonperfusion and occlusion occur in the early stages of DR (background DR) have yet to be fully characterised. Various rheological abnormalities, found in association with diabetes or diabetic animal models, are suggested to have a role in capillary non-perfusion. These include reduced retinal blood flow, ${ }^{3}$ increased plasma and whole blood viscosity, ${ }^{4}$ increased aggregation of platelets ${ }^{5}$ and erythrocytes, ${ }^{6}$ and decreased filterability of erythrocytes. ${ }^{7}$

More recently, increasing evidence is being found that indicates a crucial role for leucocytes in capillary nonperfusion. Leucocytes, which under normal conditions are 700 times less deformable than erythrocytes, ${ }^{8}$ have also been shown to have decreased filterability under diabetic conditions. ${ }^{9}$ Activated monocytes and granulocytes are found in increased numbers in the diabetic environment. ${ }^{11}$ Additionally, increased leucostasis in the retinal microvasculature is also well documented in diabetic rat models. ${ }^{10}{ }^{12-14} \mathrm{It}$ has been proposed that the diabetic environment produces a "sticky" vascular endothelial phenotype in which increased adhesion is the mechanism behind the increased leucostasis observed in the diabetic environment. Although in vivo studies, in which various adhesion molecules are blocked, have shown a significant reduction in leucostasis in experimental animal models, ${ }^{12-14}$ adhesion molecule blockade has yet to produce normal levels of leucostasis in diabetic subjects, suggesting that other mechanisms may be involved.

In this study we investigated the possibility that increased ICAM-1 expressed on the retinal microcirculation has a causatory role in the occurrence of capillary non-perfusion in human diabetic subjects. This was done through immunohistochemical analysis of frozen retinal sections from 19 eyes from 19 non-diabetic control subjects and 41 eyes from 37 diabetic people using specific antibodies against ICAM-1 as well as three additional well characterised adhesion molecules (E-selectin, P-selectin, and VCAM-1), the endothelial marker CD31 and PAL-E, a vascular leakage marker associated with diabetic retinopathy..$^{15} 16$

\section{MATERIALS AND METHODS \\ Tissue samples}

Nineteen eyes from 19 human donors with no known history of diabetes mellitus and 41 eyes from 37 known diabetic human donors (16 type 1, 18 type 2, and three unknowns) were kindly provided by the Cornea Bank, Amsterdam, after removal of corneal buttons for transplantation. A summary of the known patient data is contained in table 1. For reasons of privacy, information regarding the presence and/or degree of diabetic retinopathy was, in most cases, not available. Intact eyes were snap frozen in isopentane and stored at $-70^{\circ} \mathrm{C}$ until used. The use of human material was in accordance with the Declaration of Helsinki on the use of human material for research.

\section{Immunoperoxidase staining}

Tissue blocks containing the mid-peripheral retina and posterior pole of one frozen eye of each patient were cut

Abbreviations: $D R$, diabetic retinopathy; $E C M$, extracellular matrix; $\mathrm{GCL}$, ganglion cell layer; OLM, outer limiting membrane; ONL, outer nuclear layer; RPE, retinal pigment epithelium; RVE, retinal vascular endothelium 


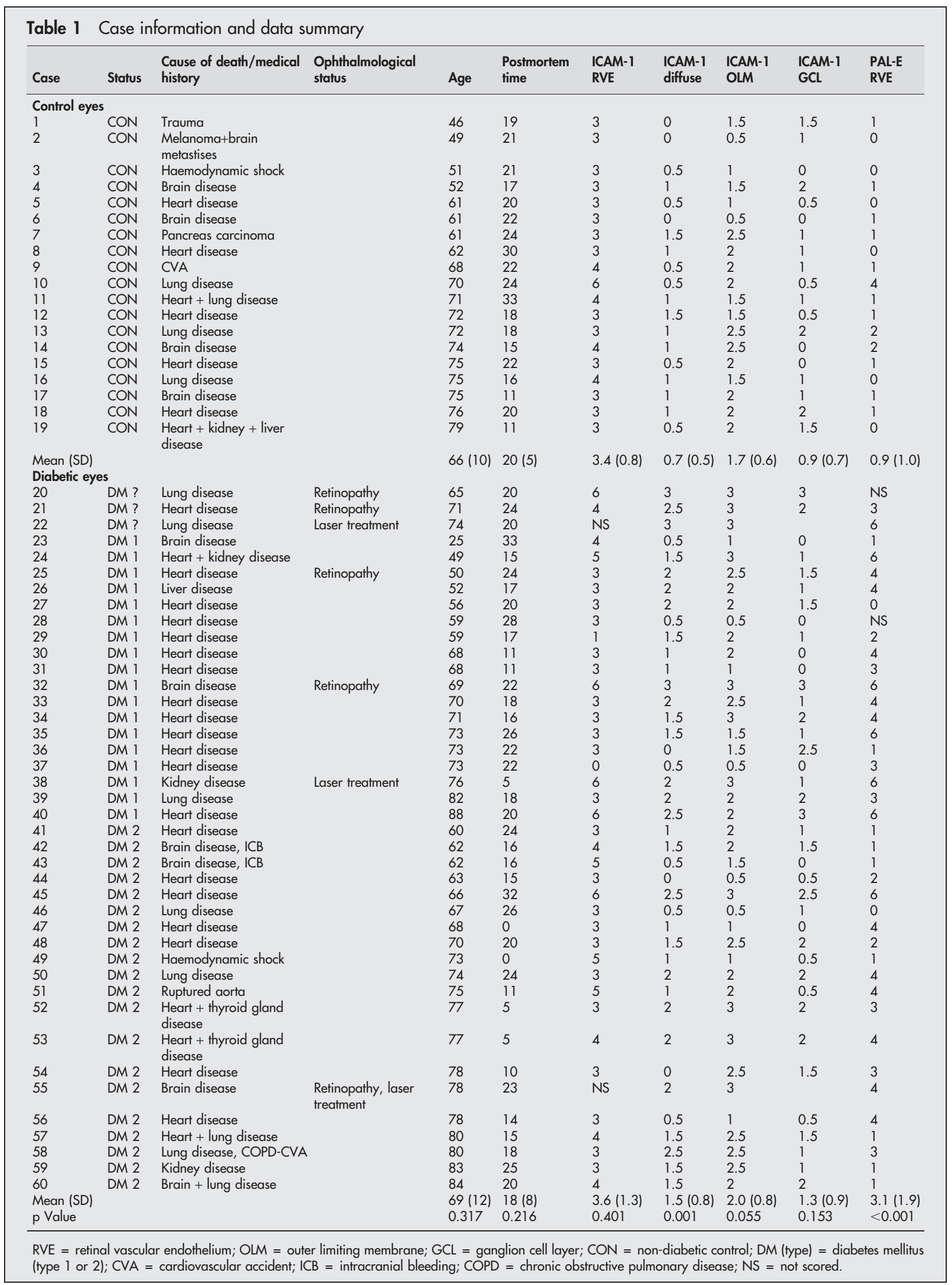

from the globes following a standard protocol. Cryosections $(10 \mu \mathrm{m})$ from tissue blocks were stained using an indirect immunoperoxidase procedure as previously described by Hofman et al. ${ }^{17}$ The sections were stained with the following antibodies: monoclonal antibodies (MAbs) ICAM-1 (84H10, Immunotech, Marseille, France and 15.2, Novocastra, Newcastle upon Tyne, UK), E-selectin (BBIg-E4, R\&D Systems, Abingdon, UK), P-selectin, VCAM-1 (American 
Type Culture Collection (ATCC), Rockville, MD, USA), and the anti-endothelial monoclonal antibodies $P A L-E^{18}$ and CD31 (EN-4, Sanbio, Uden, Netherlands). ICAM-1, Eselectin, and VCAM-1 MAbs were kindly provided by Rob Keehnen (Academic Medical Center, Amsterdam, Netherlands). Primary antibody was omitted for negative controls. Additionaly, immunohistochemistry was performed using Powervision histostaining reagents (ImmunoVision Technologies, Daly City, CA, USA) on selected sections stained for ICAM-1.

\section{Data analysis}

All sections were examined by two independent observers and scored in a masked fashion. The scores given by the observers for each eye were averaged and then used in data analysis. The distribution and intensity of staining product for PAL-E on vascular endothelium was graded semiquantitatively in the retina as follows: 0, no staining; 1, sporadic (less than four positive vessels per $5 \mathrm{~mm}$ section), weak staining; 2, sporadic, marked staining; 3, patchy (localised areas of stained capillaries, but less than $50 \%$ of vessels positive), weak staining; 4, patchy, marked staining; 5, uniform (more than 50\% of vessels positive), weak staining; 6 , uniform, marked staining. The staining product of ICAM-1 was complex with several distinct patterns observed in the retina. Three separate systems were used to grade these various patterns. Diffuse ICAM-1 staining, outer limiting membrane (OLM) ICAM-1 staining, and ganglion cell layer (GCL) perivascular ICAM-1 staining were graded by intensity on a scale from 0 to 3 as follows: 0, no staining; 1, weak; 2, moderate; 3, strong. Staining of the retinal pigment epithelium (RPE) was graded such that ICAM-1 staining on $25 \%$ or less of the RPE was considered sporadic, staining on greater than $25 \%$ but less than $75 \%$ of the RPE was considered patchy, and staining on $75 \%$ or more of the RPE was considered uniform. The ICAM-1 staining of the retinal vascular endothelium (RVE) was graded using the vascular endothelial PAL-E grading system. For E-selectin, P-selectin, and VCAM-1 the number of positively staining vessels in the retina were scored.

Statistical significance in comparisons of means between two groups was calculated using the Mann-Whitney nonparametric test for two independent samples. Two tailed $p$ values of 0.05 or less were considered significant. Correlations between parameters were determined by calculating Kendall's tau-b correlation coefficient, which is a nonparametric Spearman rank correlation, with no Gaussian

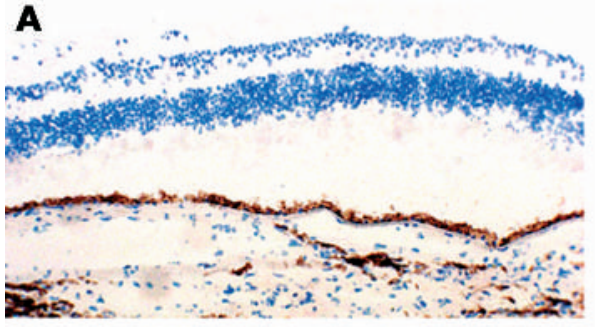

C
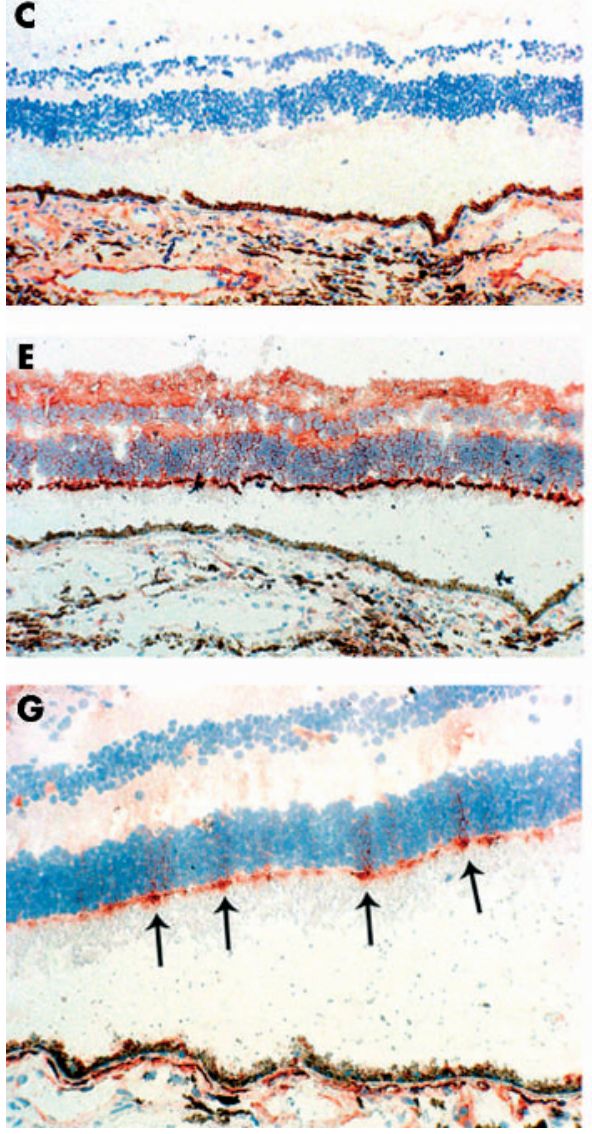

B

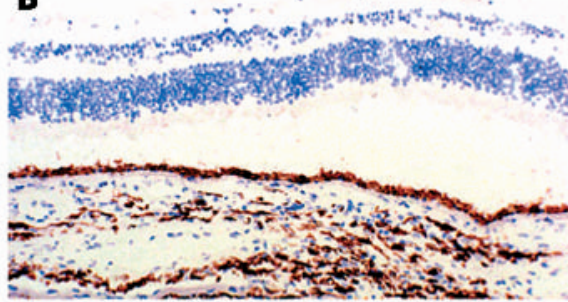

D
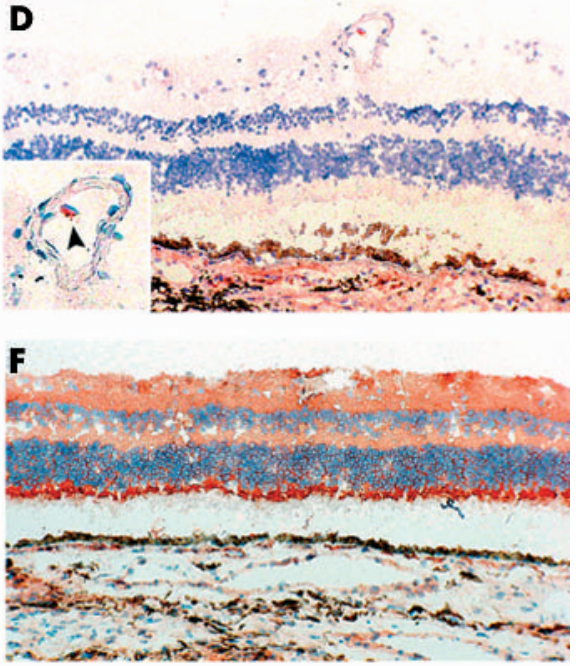

Figure 1 AEC staining of adhesion molecules VCAM-1 (A), E-selectin (B), P-selectin (C and D), and ICAM- 1 ( $E, F$, and $G$ ) in human diabetic retina. An example of platelet associated Pselectin vascular staining (arrowhead) with an inset of the large vessel; magnification $\times 125$ (D). ICAM-1 staining with clone $84 \mathrm{~h} 10$ (E) and 15.2 (F). Radial ICAM-1 staining suggesting possible Müller cells expression (G). (AF) are from same diabetic subject; magnification $\times 62.5$. $(G)$ is a separate diabetic subject; magnification $\times 120$. All retinal cryosections were counterstained with haematoxylin. 


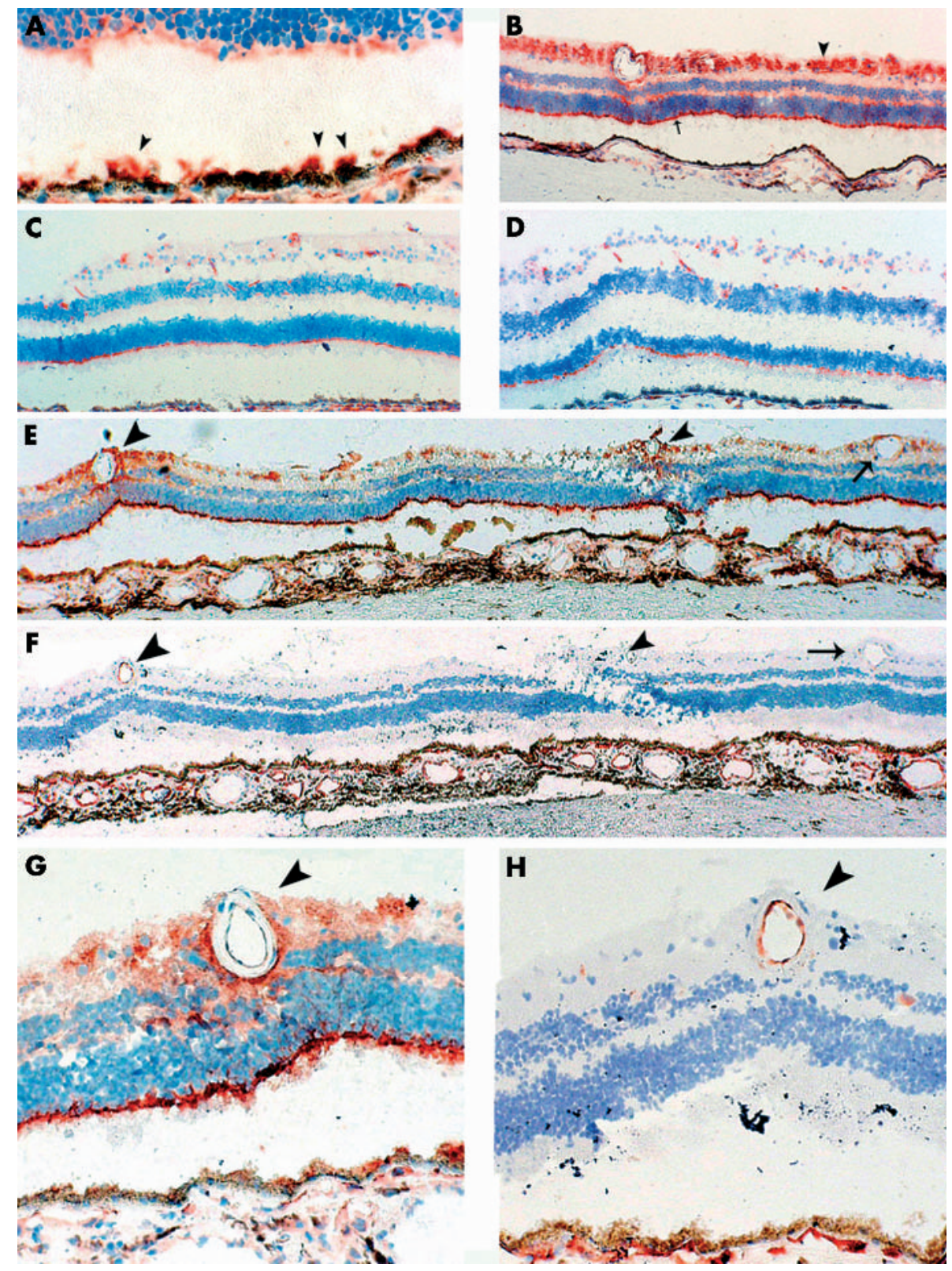

Figure 2 Patterns of ICAM-1 staining in diabetic retinas $(A, B$ and $D-H)$ and control retina (C). (A) RPE staining (tufts indicated by arrowheads):

magnification $\times 250$. (B) $G C L$ and OLM staining (arrowheads and arrows, respectively; magnification $\times 62.5$. (C and D) Vascular endothelium staining in control and diabetic subject, respectively; magnification $\times 62.5$. (E and F) Diabetic retinal cryosection stained with ICAM-1 (E) and serial cryosection stained with PAL-E (F) Note that the degree of perivascular ICAM-1 staining positively correlates with the degree of PAL-E staining in the two positive large vessels (arrowheads), while the large vessel that is negative for perivascular ICAM-1 is also PAL-E negative (arrows). This suggests that the perivascular ICAM-1 staining could be the product of soluble ICAM-1 leaked from the retinal vasculature. (E) and (F) are at $\times 50$ magnification. $(\mathrm{G})$ and $(\mathrm{H})$ Left most positive large vessel from (E) and (F) at $\times 125$ magnification. All figures show retinal cryosections stained with AEC and haematoxylin counterstaining.

assumptions, that accounts for ties. A two tailed p value was calculated to determine the statistical significance of the correlation, with $\mathrm{p}$ values of 0.05 or less considered significant.

Table 2 Distribution of vascular endothelial ICAM-1 staining scores

\begin{tabular}{|c|c|c|c|}
\hline & Control & DMI & DM2 \\
\hline \multicolumn{4}{|c|}{ RVE ICAM-1 staining } \\
\hline & & 1 & \\
\hline & & 1 & \\
\hline & & & \\
\hline & 14 & 11 & 11 \\
\hline & 4 & 1 & 4 \\
\hline & & 1 & 3 \\
\hline & 1 & 3 & 1 \\
\hline Total & 19 & 18 & 19 \\
\hline
\end{tabular}

\section{RESULTS}

VCAM-1, E-selectin, and P-selectin

There was no appreciable staining of VCAM-1 or E-selectin in retina for all retinal sections scored (fig $1 \mathrm{~A}$ and $\mathrm{B}$ ). P-selectin staining was only occasionally observed in diabetic and nondiabetic eyes (fig IC and D). This staining was often localised to the endothelium of many larger vessels present in the ganglion cell layer and appeared to be associated with the presence of platelets located within the vascular lumen (fig 1D). Sporadic staining of capillary endothelium was occasionally observed. No significant difference in P-selectin staining between the control and diabetic groups was observed.

\section{ICAM- 1}

Various patterns of ICAM-1 staining were observed in all layers of the retina with the only exception being the inner and outer segments of the photoreceptors. A fine diffuse granular ICAM-1 staining was observed in a majority of the control and diabetic eyes. This diffuse staining was mainly observed throughout the outer nuclear layer (ONL) and inner 
nuclear layer (INL) and occasionally in the inner plexiform layer (IPL) and outer plexiform layer (OPL) (fig IE). To determine if this diffuse staining was non-specific background staining, the entire series of retinal sections was stained with a second ICAM-1 clone (15.2) (fig IF). Selected retinas were also stained with ICAM-1 using the Powervision method which does not rely on the use of an avidin-biotin complex, thus avoiding non-specific staining caused by endogenous biotin (data not shown). The diffuse ICAM-1 staining remained evident for all procedures performed. Based on these findings we determined the diffuse ICAM-1 staining to be of importance and it was scored based on intensity. Additionally, some diabetic eyes displayed "stripes" of ICAM-1 staining extending radially through the retina from the OLM through the ONL (fig lG). Significantly less diffuse ICAM-1 staining was observed in the control eyes than in the diabetic eyes (0.7 (SD 0.5$)$ and 1.5 (0.8) respectively; $\mathrm{p}=0.001$ ).

A degree of ICAM-1 staining was seen on the RPE of all eyes. Owing to the pigment coloration of the epithelial cells, the staining was at times difficult to judge. Darker patches of RPE that demonstrated faint brownish-red colouring on the RPE were considered positive staining. These darker patches often occurred in a dotted line pattern along the apical periphery of the cellular surface of the RPE, proximal to the retina. In areas of strong staining ICAM-1 appeared in small tufts on the RPE cells (fig 2A). In control eyes, staining of the RPE was, on average, weak and distributed over less than half of the RPE (3.0 (1.4)). In the diabetic retinas we observed a significant $(\mathrm{p}=0.038)$ increase in intensity and distribution of staining over that seen in the RPE of the controls (3.8 (1.3)). Some degree of ICAM-1 staining was also observed along the OLM of every eye. ${ }^{14}$ In general, this appeared as a granular band of moderate staining along the entire length of the OLM (fig 2B). On average, the staining of the diabetic OLM $(2.0(0.8))$ was slightly higher than that of the controls $(1.7(0.6))$. This difference was not found to be statistically significant.

In the GCL of the retina, a perivascular pattern of ICAM-1 staining was frequently observed in both control and diabetic eyes (fig 2B). A small but not significant difference was observed in the GCL staining of control and diabetic eyes $($ mean $=0.9, \mathrm{SD}=0.7$ and mean $=1.3, \mathrm{SD}=0.9 ;$ respec tively).

Vascular endothelial staining of ICAM-1 was observed on capillaries in the INL, IPL, and GCL, and also on larger vessels in the IPL and GCL. This staining was witnessed in every control eye and all but one of the diabetic eyes. No statistically significant difference was detected between vascular endothelial staining of ICAM-1 in control and diabetic eyes, with an average score of $3.4(0.8)$ for control and 3.6 (1.3) for diabetic eyes. Figures 2C and D illustrate staining patterns observed in both control and diabetic eyes, while table 2 shows the distribution of scores for the control, diabetes type 1 , and diabetes type 2 eyes.

\section{PAL-E}

In addition to the adhesion molecule staining, PAL-E staining was performed on all retinas as an indicator of blood-retina barrier (BRB) dysfunction and microvascular leakage associated with DR. ${ }^{15}{ }^{16}$ Strong staining of the antibody PAL-E was observed in the choroid vasculature of all eyes. In the retina, PAL-E staining was mainly observed on the capillaries and, occasionally, larger vessels throughout the inner retina of diabetic eyes. Correlation coefficients (Kendall's tau-b) were calculated to investigate the relation between PAL-E and the various forms of ICAM-1 staining. Vascular endothelial PAL-E staining of the retina was found to be significantly correlated with both diffuse $(p<0.001$, $r=0.445)$ and OLM $(\mathrm{p}<0.001, r=0.415)$ ICAM- 1 staining in all eyes. Interestingly, it was also observed that diabetic eyes which displayed PAL-E staining on larger, non-capillary vessels (LVs) in the GCL $(n=15)$ had significantly higher levels of diffuse, OLM, RVE, and GCL perivascular ICAM-1 staining when compared to diabetic eyes with no LV PAL-E staining $(\mathrm{n}=24)$ (data not shown). Figures $2 \mathrm{E}-\mathrm{H}$ show an example of a strongly positive PAL-E large vessel that also demonstrates strong perivascular/diffuse staining for ICAM1 .

\section{DISCUSSION}

In this study we have shown that ICAM-1 immunoreactivity is present on the RVE of non-diabetic subjects. More importantly, we were unable to observe a significant difference in vascular endothelial ICAM-1 immunoreactivity between eyes from diabetic and non-diabetic subjects. In addition, we also observed the presence of a diffuse ICAM-1 immunoreactivity in many retinas. The intensity of this diffuse immunoreactivity varied from retina to retina and showed a significant positive correlation with the presence of diabetes and with the deterioration of the BRB as indicated by PAL-E immunoreactivity in the retina.

Using the supply of diabetic and non-diabetic ocular tissue that was at our disposal, we sought to examine the localisation of ICAM-1 in the diabetic retina compared to the non-diabetic retina. While the immunohistochemical technique used in this study is not able to quantitatively evaluate the amount or character of the ICAM-1 present in each retina, it is currently the only technique available that can reveal the localisation of ICAM-1 protein within the vascular endothelium of human retina tissue. Though not able to quantify the amount of ICAM-1 present, qualitative observations of the intensity of immunoreactivity were made in masked fashion by two independent observers.

The finding that there is no observable difference in ICAM1 immunoreactivity of the RVE between diabetic and nondiabetic subjects is in disagreement with the observations of Mcleod et al who, in a similar immunohistochemical study, observed a significant difference in vascular endothelial ICAM-1 immunoreactivity. ${ }^{11}$ In contrast with our findings, McLeod et al only rarely observed RVE immunoreactivity in control non-diabetic subjects, whereas we were able to observe RVE immunoreactivity in all control subjects. Factors such as age, cause of death, postmortem time, and enucleation time were comparable for the subject populations from both studies and, therefore, ruled out as possible causes for the differing results. A possible explanation for this discrepancy could be sampling error, as only six non-diabetic control subjects were observed by McLeod et al compared to the 19 non-diabetic control subjects observed in this study. It is also important to note that the presence of retinal vascular endothelial ICAM-1 immunoreactivity in normal human retina has also been reported in studies by Duguid et al ${ }^{19}$ and Hill et al..$^{20}$

The results found in our study also seemingly contradict the findings of recent in vivo studies on diabetic rats. ${ }^{12}{ }^{13} 21$ With a closer look, however, one finds that this is not necessarily the case. In these in vivo studies it is shown that ICAM-1 gene expression is increased in the retinas of diabetic rats and that leucostasis in the retinal vasculature is decreased after ICAM-1 blockade with a neutralising antibody. ${ }^{12}$ As the increased gene expression of ICAM-1 in the diabetic rat retinas was not isolated to the endothelium of the retina, but was found for the entire retina, it is possible that another cell type in the retina is responsible for the increased ICAM-1 gene expression observed by Miyamoto et al. ${ }^{12}$ Likewise, the increased diffuse ICAM-1 immunoreactivity in diabetic retinas observed in our study could be the end result 
of an increased ICAM-1 gene expression in the diabetic human retinas, similar to that seen in the retinas of the diabetic rats. Increased leucostasis observed in diabetic rats was shown to be reduced by $48.5 \%$ after ICAM-1 blockade ${ }^{12}$ and by $62 \%$ upon blockade of $\mathrm{CDI}^{13},{ }^{13}$ an ICAM-1 ligand present on leucocytes. Despite the blockade of ICAM-1 and one of its major leucocyte associated ligands, the leucostasis was not reduced to that observed in control rats in these studies. This, in combination with the findings of this study, could suggest that the role adhesion molecules play in increased leucostasis seen in this rat model is secondary to other pathological events related to diabetes. Some possible mechanisms primary to increased expression of adhesion molecules could be the narrowing of capillary lumens as a result of endothelial cell hypertrophy, ${ }^{17}$ increased plasma levels of the vasoconstrictor endothelin-1, ${ }^{22}$ or decreased blood flow in the retina, ${ }^{23-25}$ although recent work from Abiko et al suggests that a correlation between leucostasis and decreased blood flow does not exist in diabetic rats. ${ }^{26}$ In these situations it is feasible that even constitutive levels of ICAM-1 would be enough to adhere slower moving or partially obstructed leucocytes to the endothelium causing increased leucostasis.

It is also important to note that in these experimental studies, rats were invariably investigated only a few weeks after induction of diabetes by streptozotocin (STZ). In humans, clinical DR, and capillary non-perfusion in particular, is not observed earlier than after 7-10 years of DM. The STZ model in its early phase could best be regarded as a model of acute diabetes. Extrapolation of findings in this model to explain mechanisms involved in the long term damage to the retina in human DR may not be appropriate.

As mentioned previously, we observed an increase in diffuse ICAM-1 immunoreactivity in the retinas of diabetic eyes when compared to non-diabetic eyes. While it is not possible, on the basis of this study alone, to determine the origin of the observed increased diffuse ICAM-1 staining, there are three likely possibilities. One explanation is that a soluble form of ICAM-1, the presence of which has been shown to be increased in the serum of diabetic patients, ${ }^{27}$ leaks from the blood supply into the retina through retinal blood vessels that have lost their blood-retina barrier characteristics. Leaky vessels are characteristic of diabetic retinopathy ${ }^{2}$ and in this study we observed a correlation between the diffuse ICAM-1 immunoreactivity and the presence of retinal vessels that have lost their blood-brain barrier as measured by PAL-E immunoreactivity. ${ }^{15} 16$ The second possible explanation is that the diffuse immunoreactivity is from ICAM-1 produced by cells within the retina itself. When stimulated with tumour necrosis factor $\alpha$, rat astrocytes produce sICAM-1 through the cleavage of membrane associated ICAM- 1 by metalloproteases, ${ }^{28}$ suggesting that glial cells can serve as a source of sICAM-1 in the retina. Diffuse ICAM-1 immunoreactivity has also been observed by Verbeek et al. in senile plaques in brain tissue of patients with Alzheimer's disease. ${ }^{29}$ This diffuse ICAM-1 staining was suggested not to originate from the vasculature of the brain as the blood-brain barrier was still intact. ICAM-1 is known to bind to hyaluronan (HA), ${ }^{30}$ a constituent of the extracellular matrix (ECM) and fibrinogen, ${ }^{31}$ a serum protein which has been shown to leak into diabetic retinas. ${ }^{32}$ It is, therefore, feasible that ICAM-1 shed by glial cells bind to these molecules throughout the ECM and give rise to the diffuse immunoreactivity observed in both of these studies. Soluble ICAM-1 has been shown to act as an angiogenic factor that stimulates chemokinetic endothelial cell migration, endothelial cell differentiation and vessel sprouting in vitro and angiogenesis in vivo. ${ }^{33}$ The presence of sICAM-1 could be indicative of a pro-angiogenic environment in the retina and, therefore, have an important role in the pathogenesis of diabetic retinopathy.

It is also feasible that the diffuse ICAM- 1 staining, and that observed along the OLM, is the result of membrane associated ICAM-1. The striped ICAM-1 ONL staining observed in several diabetic eyes has a pattern that suggests it could be associated with Müller cells. As the Müller cells are present throughout the retina and stretch radially from the OLM to the ILM, should they become activated and upregulate ICAM-1 over their entire length, they could produce an ICAM-1 staining comparable to the diffuse ICAM-1 staining observed in this study. As the end feet of Müller cells are a main component of the OLM they are also a possible source of the OLM ICAM-1 staining observed.

In conclusion, in this observational study we were unable to find evidence of a "sticky" phenotype, common to vasculature in inflamed tissue, in the retinal vasculature of diabetic subjects. Of the four major molecules involved in leucocyte adhesion that we examined in this study-namely, E-selectin, P-selectin, VCAM-1, and ICAM-1, we observed no increase in retinal vascular expression in diabetic subjects when compared to controls. This suggests that the pathologies related to diabetic retinopathy are not caused by classic inflammation and that leucocyte adhesion via ICAM-1, while probably playing a part, is unlikely to be the primary cause of leucostasis and capillary occlusion in humans. In addition, we found evidence of what appears to be non-endothelial associated ICAM-1 in the retina, the levels of which correlate highly with the presence of diabetes. Further studies will be needed to determine the primary cause of capillary occlusion in diabetic retinopathy and to investigate further the presence of non-endothelial ICAM-1 and the implications it may have in the pathology of diabetic retinopathy.

\section{ACKNOWLEDGEMENTS}

Supported by Diabetes Fonds Nederland (Grant 98.131) and the Edward en Marienne Blaauw Fonds.

The authors wish to thank Professor S Pals for supplying antibodies and helpful comments.

\section{Authors' affiliations}

J M Hughes, A Brink, A N Witmer, M Hanraads-de Riemer, I Klaassen, R O Schlingemann, Ocular Angiogenesis Group, Department of Ophthalmology, Academic Medical Center, University of Amsterdam, Amsterdam, Netherlands

\section{REFERENCES}

1 Aiello LP, Gardner TW, King GL, et al. Diabetic retinopathy. Diabetes Care 1998;21:143-56.

2 Garner A. Histopathology of diabetic retinopathy in man. Eye 1993;7(Pt 2):250-3.

3 Konno S, Feke GT, Yoshida A, et al. Retinal blood flow changes in type I diabetes. A long-term follow-up study. Invest Ophthalmol Vis Sci 1996;37:1140-8

4 Kurose I, Anderson DC, Miyasaka M, et al. Molecular determinants of reperfusion-induced leukocyte adhesion and vascular protein leakage. Circ Res 1994:74:336-43

5 Sagel J, Colwell JA, Crook L, et al. Increased platelet aggregation in early diabetes mellitus. Ann Intern Med 1975;82:733-8.

6 Schmid-Schonbein H, Volger E. Red-cell aggregation and red-cell deformability in diabetes. Diabetes 1976;25:897-902.

7 McMillan DE, Utterback NG, La Puma J. Reduced erythrocyte deformability in diabetes. Diabetes 1978;27:895-901.

8 Chien S, Schmalzer EA, Lee MM, et al. Role of white blood cells in filtration of blood cell suspensions. Biorheology 1983;20:11-27.

9 Miyamoto K, Ogura Y, Kenmochi S, et al. Role of leukocytes in diabetic microcirculatory disturbances. Microvasc Res 1997;54:43-8.

10 Schröder S, Palinski W, Schmid-Schönbein GW. Activated monocytes and granulocytes, capillary nonperfusion, and neovascularization in diabetic retinopathy. Am J Pathol 1991;139:81-100.

11 McLeod DS, Lefer DJ, Merges C, et al. Enhanced expression of intracellular adhesion molecule-1 and P-selectin in the diabetic human retina and choroid. Am J Pathol 1995; 147:642-53.

12 Miyamoto K, Khosrof S, Bursell S, et al. Prevention of leukostasis and vascular leakage in streptozotocin-induced diabetic retinopathy via intercellular adhesion molecule-1 inhibition. Proc Natl Acad Sci USA 1999;96:10836-41. 
13 Barouch FC, Miyamoto K, Allport JR et al Integrin-mediated neutrophil adhesion and retinal leukostasis in diabetes. Invest Ophthalmol Vis Sci 2000;41:1153-8.

14 Joussen AM, Murata T, Tsujikawa A, et al. Leukocyte-mediated endothelial cell injury and death in the diabetic reting. Am J Pathol 2001:158:147-52.

15 Schlingemann RO, Hofman P, Vrensen GF, et al. Increased expression of endothelial antigen PAL-E in human diabetic retinopathy correlates with microvascular leakage. Diabetologia 1999;42:596-602.

16 Schlingemann RO, Hofman P, Anderson L, et al. Vascular expression of endothelial antigen PAL-E indicates absence of blood-ocular barriers in the normal eye. Ophthalmic Res 1997;29:130-8.

17 Hofman P, van Blijswijk BC, Gaillard PJ, et al. Endothelial cell hypertrophy induced by vascular endothelial growth factor in the retina: new insights into the pathogenesis of capillary nonperfusion. Arch Ophthalmol 2001;119:861-6.

18 Schlingemann RO, Dingjan GM, Emeis JJ, et al. Monoclonal antibody PAL-E specific for endothelium. Lab Invest 1985;52:71-76.

19 Duguid IG, Boyd AW, Mandel TE. The expression of adhesion molecules in the human retina and choroid. Aust N Z J Ophthalmol $1991 ; 19: 309-15$.

20 Hill TA, Stanford MR, Graham EM, et al. A new method for studying the selective adherence of blood lymphocytes to the microvasculature of human retina. Invest Ophthalmol Vis Sci 1997;38:2608-18.

21 Miyamoto K, Ogura Y. Pathogenetic potential of leukocytes in diabetic retinopathy. Sem Ophthalmol 1999;14:233-9.

22 Takahashi K, Ghatei MA, Lam HC, et al. Elevated plasma endothelin in patients with diabetes mellitus. Diabetologia 1990;33:306-10.

23 Feke GT, Buzney SM, Ogasawara H, et al. Retinal circulatory abnormalities in type 1 diabetes. Invest Ophthalmol Vis Sci 1994;35:2968-75.
24 Bursell SE, Clermont AC, Kinsley BT, et al. Retinal blood flow changes in patients with insulin-dependent diabetes mellitus and no diabetic retinopathy. Invest Ophthalmol Vis Sci 1996;37:886-97.

25 Clermont AC, Aiello LP, Mori F, et al. Vascular endothelial growth factor and severity of nonproliferative diabetic retinopathy mediate retinal hemodynamics in vivo: a potential role for vascular endothelial growth factor in the progression of nonproliferative diabetic retinopathy. Am J Ophthalmol 1997; 124:433-46.

26 Abiko T, Abiko A, Clermont AC, et al. Characterization of retinal leukostasis and hemodynamics in insulin resistance and diabetes: role of oxidants and protein kinase-C activation. Diabetes 2003;52:829-37.

27 Olson JA, Whitelaw CM, McHardy KC, et al. Soluble leucocyte adhesion molecules in diabetic retinopathy stimulate retinal capillary endothelial cell migration. Diabetologia 1997:40:1156-71.

28 Lyons PD, Benveniste EN: Cleavage of membrane-associated ICAM-1 from astrocytes: involvement of a metalloprotease. Glia 1998;22:103-12.

29 Verbeek MM, Otte-Holler I, Westphal JR, et al. Accumulation of intercellular adhesion molecule- 1 in senile plaques in brain tissue of patients with Alzheimer's disease. Am J Pathol 1994;144:104-15.

30 McCourt PA, Ek B, Forsberg N, et al. Intercellular adhesion molecule-1 is a cell surface receptor for hyaluronan. J Biol Chem 1994;269:30081-4.

31 Duperray A, Languino LR, Plescia J, et al. Molecular identification of a novel fibrinogen binding site on the first domain of ICAM-1 regulating leukocyteendothelium bridging. J Biol Chem 1997;272:435-41.

32 Murata T, Ishibashi T, Inomata H. Immunohistochemical detection of extravasated fibrinogen (fibrin) in human diabetic retina. Graefes Arch Clin Exp Ophthalmol 1992;230:428-31.

33 Gho YS, Kleinman HK, Sosne G. Angiogenic activity of human soluble intercellular adhesion molecule-1. Cancer Res 1999:59:5128-32. 